$$
\text { Optical Engineering }
$$

SPIEDigitalLibrary.org/oe

\title{
OE 2013 List of Reviewers
}


Optical Engineering would like to sincerely thank the following individuals who served as reviewers in 2013. The success of our publication hinges on the voluntary contributions of time and energy put forth by these professionals.

\begin{tabular}{|c|c|}
\hline Ahmed Abd El-Latif & Marco Anisetti \\
\hline Mohd Kamil Abd Rahman & Jean-Pierre Antoine \\
\hline Mohamed Abdel Rahman & Dan Apostol \\
\hline Fatma Abdelkefi & Roger Appleby \\
\hline Ruzairi Abdul Rahim & Vyacheslav Aranchuk \\
\hline Ibrahim Abdulhalim & Josep Arasa Marti \\
\hline Ghaleb Abdulla & Helder Araujo \\
\hline Fairuz Abdullah & Jonathan Arenberg \\
\hline Jaafar Abdullah & Mark Arend \\
\hline Mohammad Faiz Liew Abdullah & Justo Arines \\
\hline Abhijat Abhyankar & Gamini Ariyawansa \\
\hline Mehrdad Abolbashari & John W. Arkwright \\
\hline Gil Abramovich & Errico Armandillo \\
\hline Sofia Acosta-Ortiz & Robert Arnone \\
\hline Mariana Adam & Rameez Asif \\
\hline Athos Agapiou & Lahsen Assoufid \\
\hline Eyal Agassi & Vasily Astratov \\
\hline Farzin Aghdasi & George Atanasoff \\
\hline Esmail Ahouzi & Peter A. Atanasov \\
\hline Md. Shamim Ahsan & Paul Atcheson \\
\hline Jun Ai & Manik Attygalle \\
\hline Bruno Aiazzi & Oscar Au \\
\hline Devrim Akca & Mathieu Aubailly \\
\hline Moulay Akhloufi & Jason Auxier \\
\hline Frank Akins & Yasuhiro Awatsuji \\
\hline Maki Akioka & Tayfun Aytac \\
\hline Muhammad Nadeem Akram & Axel Baeuerle \\
\hline Mohammad S. Alam & Jing Bai \\
\hline Manal Alammari & Antonio Baldi \\
\hline Tapani Alasaarela & Anders Ballestad \\
\hline Ana-Marial Albu & Janis Balodis \\
\hline Adrian Alexandrescu & Giorgio Balzarotti \\
\hline Jalil Ali & Neal Bambha \\
\hline Syed Zafar Ali & Nikos Bamiedakis \\
\hline Khaled Aljasem & Victor A. Banakh \\
\hline Frederic Allegrini & Sumith Bandara \\
\hline Monica Allen & Saswatee Banerjee \\
\hline Thomas Allsop & Curtis E. Banks \\
\hline Jose Almeida & Francesco Banterle \\
\hline Guillaume Alombert Goget & Paul Bao \\
\hline Miguel A. Alonso & Qiaoliang Bao \\
\hline Zahir Alpaslan & Yuan Bao \\
\hline Jose Alvarez-Chavez & Jose Baptista \\
\hline Nicolas Alvertos & James Barabas \\
\hline Fabio Alves & Meir Barak \\
\hline Ariel Amato & Nicolas R. Barbeau \\
\hline Pierre Ambs & Sergio Barbero \\
\hline Ruhul Amin & Eduardo Barbosa \\
\hline Junming An & Alessandro Barducci \\
\hline Sangeetha Anandan & Peter Baricholo \\
\hline Gholamreza Anbarjafari & Boris Barmashenko \\
\hline Torben E. Andersen & Kenneth J. Barnard \\
\hline Paulo Andre & Bernardino Barrientos \\
\hline S. Michael Angel & Ricardo Barrios \\
\hline George Angeli & Michael Bass \\
\hline Elli Angelopoulou & Paolo Bassi \\
\hline Oleg Angelsky & Prashant Baveja \\
\hline
\end{tabular}

Yahya Baykal

Guy Beadie

Romeo Beccherelli

Laurent Bechou

Martin Becker

Anish Bekal

Augusto Belendez

Tomas Belenguer

Mikhail Belenkii

W. Bryan Bell

C. Fabian Benitez-Quiroz

Alain Bensoussan

David Benton

David M. Berfanger

Erich Berloffa

Dainet Berman-Mendoza

Simone Berneschi

Basanta Bhaduri

Shivakiran Bhaktha B.N.

Kamaljit Bhatia

Shanti Bhattacharya

Ujjal Bhowmik

Paolo Bianchini

Francesco Bianconi

Bernd Bickel

Justin Bickford

Leila Birolo

Joerg Bischoff

Nrusingh Biswal

Abhijit Biswas

Anjan Biswas

Nikita Bityrin

Hans Bjelkhagen

Stephen Black

John Black, Jr.

Erlan Bliss

Liu Bo

Andrew Bodkin

Matthew Bohn

Klaus Bohnert

Marco Bonaglia

Donald Bone

Glenn Boreman

Dibio Borges

Krzysztof Borzycki

Jeremy Bos

Ignacio Bosch Roig

Frank Bossen

Yann Boucher

Abdel-Ouahab Boudraa

Hugo Boujut

Brigitte Boulard

Henri Bouma

Salah Bourennane

Don Bouwhuis

Belloui Bouzid

V. Michael Bove, Jr. 
David Brady

John Brady

David H. Brainard

Adriana Brancaccio

Patrice Brault

Michael Bray

Terry Brennan

James Brennan III

Matthew Brennesholtz

Jeremy Brown

Steven R.J. Brueck

Andreas Brueckner

Luigi Bruno

Peter Bryanston-Cross

Richard Buchroeder

Edward Buckley

Leonard Buckley

Ryszard Buczynski

Nadezhda Bulgakova

Gerald Buller

Robert Bunch

James Burge

Julien Burgin

Ludovic Burgnies

David Burnett

Stephen Burns

W. Robert Burns

John Burris

Neal Butler

Jan Buytaert

Miguel Cabezon

Elsa Caetano

Ibrahim Cahit

Yangjian Cai

Stephen Cain

Darran R. Cairns

Antonino Cala' Lesina

Paulo Caldas

Kenneth Camilleri

Stefania Campopiano

Juan Campos

Leifeng Cao

Liangcai Cao

Alessandro Capra

Jean Carlos Cardozo da Silva

Arthur Carrieri

Rafael Carrillo

Webster Cash

Roberto Casini

William Cassarly

James Castracane

J. Michael Cathcart

Massimo Cazzanelli

Mehmet Celenk

Mario Chacon-Murguia

Biswajit Chakraborty

Rajib Chakraborty

Alex Chan

Francis Chan

Kin Chan
Gary Chanan

Vinod Chandra

Ching-Hung Chang

Horng Chang

Hyun Sung Chang

Shengjiang Chang

Gaël Chareyron

Mikhail Charnotskii

Tom Charrett

Monish Chatterjee

Siddharth Chauhan

Brahim Chebbi

Rama Chellappa

Yanne Chembo

Enqing Chen

Fei Chen

Gang Chen

Hongwei Chen

Hui-Chi Chen

Jian Chen

Jian Chen

Lawrence Chen

Ming Chen

Ming-Yang Chen

Minghua Chen

Nan-Kuang Chen

Ray Chen

Wang Chen

Weibiao Chen

Wen Chen

Xin Chen

Xu Chen

Yanxue Chen

Yihong Chen

Ying Chen

Zhihao Chen

Zhixin Chen

Chieh-Jen Cheng

Dewen Cheng

Hao Bo Cheng

Hsu-Chih Cheng

Yuen Yap Cheng

Vien Cheung

Hao Chi

Mingjun Chi

Nan Chi

Sien Chi

Yu-Chieh Chi

John Y. Chiang

Kin Seng Chiang

Andrea Chiappini

Alessandro Chiasera

Dan Chicea

Kai-Yi Chin

Arkadi Chipouline

Krishnan Chitra

Yi-Jen Chiu

Seungryong Cho

Byong-Deok Choi

Hongsik Choi
Jin Choi

Kang-Sun Choi

Ken Choi

Kwong-Kit Choi

Kyu-Hwan Choi

Sang Choi

Seong Soo Choi

Jozef Chovan

C. W. Chow

M. I. Sakib Chowdhury

Finn Christensen

Steven Christesen

Steven D. Christesen

Krzysztof Chrzanowski

Taerin Chung

James Churnside

Caterina Ciminelli

Giuseppe Cirino

Mark Clampin

Adolfo Cobo

Brandon Cochenour

Johanan Codona

Luis Coelho

Neil Collings

Mireille Commandre

Davide Comoretto

Isaac Compean

David Content

Alejandro Cornejo-Rodriguez

William Cornette

Giulio Cossu

Manuel Costa

Melissa Cote

Francois Courvoisier

Cary Cox

Edward Coyne

Julia Craven-Jones

Jared Crochet

Javier Cruz-Mota

Francisco Cuevas

Haihua Cui

Yudong Cui

Brian Cullum

Brian Culshaw

Paul Cunnigham

Anna Cysewska-Sobusiak

Moises Cywiak

Antonella D'Orazio

Anabela Da Silva

Razvan Dabu

Xuewu Dai

Fraser Dalgleish

Gian-Franco Dalla Betta

Gavin Dalton

Victor Damian

Xiaoyu Dang

Gautam Das

Kamal Das

Partha Pratim Das

Panos Datskos 
Christian David

Philip David

Frederic M. Davidson

Abundio Davila

Christopher Davis

Ian Day

Bráulio de Albuquerque

Juan de Dios

Jolyon De Freitas

Arie de Jong

Alfredo de Rossi

Russell De Young

Alessandro Deana

Pierluigi Debernardi

Alberto deCastro

Arthur J. Decker

Claire Deeb

Jason Deibel

Walter Delashmit

Francesco Della Corte

Nazif Demoli

Maciej Dems

Bing Deng

Huaxia Deng

Parag Deotare

Foued Derraz

Tolis Deslis

Vincent Despiegel

Patrick Destain

Nathalie Destouches

Susana Deustua

Nicholas Devaney

Harbans Dhadwal

Kevin Diao

Thomas Dickens

Fred Dickey

Judith Dijk

Thomas E. Dillon

Don Dilworth

Valeria Dimastrodonato

Panfeng Ding

Ran Ding

Yujie Ding

Denis Dion

Mamadou Diop

Albert Dipanda

Joris Dirckx

Sudhir Dixit

George Dobre

Lawrence Domash

Bo Dong

Hao Dong

Hui Dong

Xinyong Dong

Yi Dong

Yongkang Dong

Sarah Dooley

Pallavi Doradla

Friedhelm Dorsch

Leonid Doskolovich
Anastasios Doulamis

Pascal Doumalin

Ed Dowski

Thomas Dresel

Kyle Drexler

Dave Driscoll

Oliver Dross

Guillaume Druart

Xiaoming Duan

Adam Dubis

Yuri Dubnishchev

Alfredo Dubra

Erik Duerr

Daniel Duffin

Stefan Duffner

Wolfgang Dultz

Bradley D. Duncan

Michael Duncan

Nicholas Durr

Claire Duverger Arfuso

Patrick Earhart

Kert Edward

Oleg Efimov

Bodo Ehlers

Natalja Eikje

Michael T. Eismann

Andrew Ekpenyong

Sahar El-Naggar

Ramasamy Elavarasan

Hatem EIGhandoor

Tawfig Eltaif

Christoph Englert

Francesco Enrichi

Tae Eom

Bahadir Ergun

Maria Erro

Reiner Eschbach

Diego Esparza

Rafael Espinosa-Luna

Julio Estrada

Andreas Ettemeyer

Gary Euliss

Bruce Evans

Halil Eyyuboglu

Hilal Fadhil

Matthias Fahland

Yeshaiahu (Shaya) Fainman

Mohammad Faisal

Claas Falldorf

Zhigang Fan

Shuai Fang

Xianyong Fang

Yong Fang

Yun-tuan Fang

Analucia Vieira Fantin

Esmaeil Faramarzi

Joyce Farrell

Nathan Farwell

Gilbert Feke

Kan Feng
Xinhuan Feng

Yutao Feng

James Fergusson

Alicia FernÆndez-Oliveras

Javier Fernandez

Jose A. Ferrari

Maurizio Ferrari

Pietro Ferraro

Robert Fiete

Anthony Filippi

Steven Fiorino

Erin Fleet

Gordon Flockhart

Jorge Flores

Costel Flueraru

Richard Flynn

Faruk Fonthal

Richard Forber

Andrew Forbes

Joseph Ford

Josep Forest

Michele Fornaciari

David Forrai

Scott Foster

Augustus Fountain III

Georges Fournier

Dmitri Foursa

Eric Fox

Aaron Freeman

Marcio Freitas

Ari T. Friberg

Dale Fried

Hongyan Fu

Richard Fu

Xianping $\mathrm{Fu}$

$\mathrm{Yu} \mathrm{Fu}$

Takashi Fujii

Takeshi Fujisawa

Tetsuo Fukuchi

Herbert Funsten

Orges Furxhi

Salvador Gabarda

Davide Gadia

Ramesh Galigekere

Laurent Gallais

Almantas Galvanauskas

Angarai Ganesan

Pranabendu Ganguly

Chunqing Gao

Guangjun Gao

JianRong Gao

Liang Gao

Long Gao

Wei Gao

Xinbo Gao

Yaming Gao

Zhan Gao

Zhishan Gao

Alejandro Garcia

Pascuala Garcia-Martinez 
Carlos Garcia-Meca

Vincent Garrel

Francesca Gasparini

Daniel Gauthier

Dilip Gayen

Joseph Geary

Thomas Geernaert

Bernd Geh

Michael Gehm

Kent Geib

Victor Genberg

Nicholas George

Georgi Georgiev

Aaron Gerace

Bojan Gergic

David Geronimo

Duane Geruschat

Roland Geyl

Farzan Ghauri

Anjan Ghosh

Mher Ghulinyan

Daniel Gibson

Alex Gilerson

Richard Gill

David Gillis

Harilaos Ginis

Vincent Ginis

Maria Giraldi

Branko Glisic

Nick Glumac

Jens Gobrecht

Toon Goedeme

Arnold Goldberg

Peter Goldstein

Armando Gomez-Vieyra

Luiz Goncalves

Igor Goncharenko

Mufei Gong

Robert A. Gonsalves

Consuelo Gonzalo-Martin

Keith Goossen

Sai Siva Gorthi

Arthur Goshtasby

Nobuo Goto

Pierre Gouton

Martin Grabner

Sheryl M. Gracewski

Fremin Granados Salomon

Marco Grande

Armin Grasnick

G. Groot Gregory

Ulf Griesmann

Michael Gritz

Dan Grois

Marcus Grosse

Roger Groves

Corina Gruescu

Vitaly Gruzdev

Jason Gu

Wei Gu
Xijia Gu

Bai-ou Guan

Ugur Gudukbay

Rui Guerra

Feng Guo

Hanming Guo

Hong Guo

Huanqing Guo

Lei Guo

Xiaowei Guo

Igor Guryev

Diego Gutierrez

Julio C. Gutierrez Vega

Jay Hackett

Joonku Hahn

Frederick Haibach

Charles J. Hailey

Tobias Haist

Han Haitjema

Yaroslav Halchenko

Carl Halford

Jennifer Halman

Manfred Hammer

Philip Hammer

Dominique Hamoir

Muhammad Hamza

Jae-Ho Han

Jong-Ki Han

Ming Han

Xiang Han

Xiuyou Han

Young-Geun Han

Yukun Han

Randolf Hanke

Steen Hanson

Shu-Sheng Hao

Xiang Hao

Xiaotao Hao

Kevin Harding

Nicholas Hardy

John Harlander

Davis Hartman

Hans Hartnagel

Alexander Hartung

Stephen Harwin

Dihan Hasan

Md. Imran Hasan

Milos Hasan

Feras Hasoon

Firas Hassan

Paul O. Haugsjaa

Michael Hayford

Lakshminarayan Hazra

Jing $\mathrm{He}$

Jingliang $\mathrm{He}$

Ping'an $\mathrm{He}$

Wen $\mathrm{He}$

Y.M He

Yong $\mathrm{He}$

Zhan $\mathrm{He}$
Zhe He

John Healy

Michael Heaven

Joachim Hein

Hamid Hemmati

Karen Hendrix

Markus Henriksson

Alois Herkommer

Charles Hester

R. Hicks

Anne Hildenbrand

Koji Hirano

Harish Hiriyannaiah

Salasiah Hitam

Van Hodgkin

Bernhard J. Hoenders

Maarten Hogervorst

Timothy Holzheimer

Hyunki Hong

Seppo Honkanen

Ming-Huwi Horng

Nadir Hossain

Amir Hosseini

Sujuan Hou

Zhanjia Hou

Thomas Hough

Michael Houlne

Joseph Howard

Peter Howarth

Christopher Howell

Cheng-Chih Hsu

Paul Hsu

Wei-Feng Hsu

Rong $\mathrm{Hu}$

Wenbin $\mathrm{Hu}$

Zhaoli Hu

Zhixiong $\mathrm{Hu}$

Huang Hua

Nan Hua

Ding-Wei Huang

Jen-Fa Huang

Jie Huang

Kuo Huang

Shih-Chia Huang

Yanbo Huang

Yuanhao Huang

Yuewang Huang

John Hubbs

Merav Huber

Gary Hughes

Jean Pierre Huignard

Michael Hunt

Saleh Hussin

Hone-Ene Hwang

Sheng-Kwang Hwang

Taek Yong Hwang

Yong Seok Hwang

Youngbae Hwang

Kwangik Hyun

Baldemar Ibarra-Escamilla 
Khan Iftekharuddin

Francisco Imai

Masaharu Imaki

Mario lodice

Bogdan lonescu

John Irvine

Mohammed Nazrul Islam

Tomoyoshi Ito

Mile Ivanda

Afthab Jabeena

J. Bianca Jackson

Eddie Jacobs

Jake Jacobsen

Nicolas Javahiraly

Donald Jennings

Lars Jensen

Rajan Jha

Xiaoling Ji

Dagong Jia

Dongfang Jia

Xiao-hua Jian

Jianliang Jiang

Jinwei Jiang

Lan Jiang

Chao Jin

Guofan Jin

Wei Jin

Xiaomin Jin

Zhongliang Jing

Takahisa Jitsuno

Pierre-Marc Jodoin

Charles Joenathan

Steven E. Johnson

Tim Johnson

Joachim Jonuscheit

Nicholas Jordache

Yue-Dar Jou

Yves Jourlin

Bernard Journet

Romuald Jozwicki

Juan Juarez

DongSan Jun

Yong Ju Jung

Suganda Jutamulia

Niels König

Hideki Kakeya

Shoji Kakio

Kourosh Kalantar Zadeh

Hypolito Kalinowski

Andrew Kalukin

Canan Kamacioglu

Mohammad Kamal

Janis Kaminskis

Andrey Kanaev

Kenichi Kanatani

Paul Kane

Dong-Joong Kang

DongKyun Kang

Li-Wei Kang

Moon Gi Kang
C. C. Kankelborg

Karim Karim

Raman Kashyap

Xizheng Ke

Debbie Kedar

Edmund J. R. Kelleher

Damien Kelly

Mike Kemp

Ani Khachatrian

Diaa Khalil

Osama Khalil

Jesmin Khan

Muhammad Saeed Khan

Nasrullah Khan

Yousaf Khan

Nima Khatibzadeh

Mohammed Lamine Kherfi

Alexander Khmaladze

Ali Khounsary

Hagyong Kinm

Ajung Kim

Byung-Gyu Kim

Chang-Bong Kim

Dae Wook Kim

Daesuk Kim

Do Nyeon Kim

Dong-Wook Kim

Eung Soo Kim

Hee-Je Kim

Hoon Kim

Hwi Kim

Jae-Gon Kim

Jaisoon Kim

Jihwan Kim

Jintae Kim

Jinwoong Kim

Jong-Ok Kim

Jungwon Kim

Kwang-Taek Kim

Myun-Sik Kim

Nam Kim

Seok-min Kim

Seung-Woo Kim

Sungho Kim

Sean King

Motoi Kinoshita

Sven Kiontke

Detlef Kip

Zsolt Kiraly

Paul Kirkby

Peter Klar

Karl Klett

Mariusz Klimczak

Stanislaw Klosowicz

Michael Knaup

Joachim Knittel

George Knopf

Sergey Kobtsev

H. Kocer

Sophie Kohler
Stanley Kohn

Takuji Koike

David Koltick

Lisimachos Kondi

Qing-Jie Kong

Nikolaos Konofaos

Philipp Kornreich

Sergiy Korposh

R. John Koshel

Mahendra Kothiyal

Peter Kottke

Levente Kovacs

Yuichi Kozawa

Janusz Kozlowski

Michael Krainak

Iztok Kramberger

Volodymyr Kruglyak

Tadeusz Kryszczynski

Jakub Krzeslowski

Cuifang Kuang

Kenny Kubala

Toshiro Kubota

Dan Kuchta

Michael Kudenov

Sergey Kudryashov

Nickolai Kukhtarev

Naveen Kulkarni

Ajay Kumar

Arvind Kumar

B.V.K. Kumar

Santosh Kumar

V.P.Sudeep Kumar

Pathik Kumbhakar

R Sanjeev Kunte

Roderick R. Kunz

Toshiaki Kuri

Marco La Cascia

Antoine Labeyrie

Jean Lacoursiere

Hsin-Yi Lai

Kuo-Hsiang Lai

Puxiang Lai

Jyrki Laitinen

Fabrizio Lamberti

Xinwei Lan

Mordechai Lando

Benjamin Lane

Giovanni Laneve

Junwei Lang

Eli Lansey

Anatoliy Lapchuk

James Larimer

Fredrik Laurell

Oleg Lavrentovich

George N. Lawrence

Franz Leberl

Byoungho Lee

Byung-Uk Lee

Chih-Kung Lee

Ching-Ting Lee 
Hanlim Lee

Jen Chun Lee

Kwanghoon Lee

Sin-Doo Lee

Suk-Ho Lee

Sukhan Lee

Y. W. Lee

Yong-Gu Lee

Yun Gu Lee

Dominique Leger

Paul Leisher

James Leitch

Jinsong Leng

Richard Lepkowicz

Dan Lester

Changsheng Li

Dong Li

Fenghua Li

Guifang Li

Hongqiang $\mathrm{Li}$

Hui Li

Jianping Li

Jie Li

Jingqiang $\mathrm{Li}$

Juhao Li

Liwei Li

Peng Li

Rong-Rong Li

Shu-ping Li

Wei Li

Wei Li

Xiang Li

Xiaohui Li

Xudong Li

Yaguo Li

Yajun Li

Yan Li

Youfu Li

Yuan-Xiang Li

Yuelin Li

Zhen-hua Li

Jinyang Liang

Yanbiao Liao

Barry Lienert

Jens Limpert

Chang-Yi Lin

Chi-Yuan Lin

Chinlon Lin

Gong-Ru Lin

Guo-Shiang Lin

Kuang Tsan Lin

Shiuan Huei Lin

Wuu-Wen Lin

Norbert Lindlein

Lars Lindvold

Gordon Little

Aiqun Liu

Anqing Liu

Baiyang Liu

Bo Liu
Cheng Liu

Chenguang Liu

Deming Liu

Duan Liu

Fei Liu

Hongzhan Liu

Jian Liu

Jianguo Liu

Jung-Ping Liu

Ke Liu

Lei Liu

Mingtao Liu

Peng Liu

Shutian Liu

Wen-Fung Liu

Xiaoping Liu

Xueming Liu

Yange Liu

Yong Liu

Yuexin Liu

Zhengjun Liu

Zhishen Liu

Glynn Lloyd

Jurgen Lobert

Stephan Logunov

Hans-Peter Loock

Doug Looze

Daniel Lopez

Jose Miguel Lopez-Higuera

J. Carlos Lopez-Vazquez

Shuqin Lou

Peter Love

Heard Lowry

Vadim Lozovoy

Chao Lu

Dan Lu

Guo-Wei Lu

Jian Lu

Rong-Sheng Lu

Xuejun Lu

Yajun Lu

Yan-qing Lu

Esteban Luna-Aguilar

Li Luo

Ronnier Luo

Xian-gang Luo

Jiangtao Lv

Qiang Lv

Igor L. Lyubchanskii

Andy Jinhua Ma

Bin Ma

Haotong Ma

Jun $\mathrm{Ma}$

Ming Ma

Shaozhen Ma

Shih-Hsin Ma

Suodong Ma

Mark Mackenzie

Nicholas Madamopoulos

Joji Maeda
Daniel Magalhaes

Simon Magarill

Robert Magnusson

Lori Magruder

Virendra Mahajan

Joseph Mait

Arun Majumdar

Taron Makaryan

Zacarias Malacara

Daniel Malacara-Doblado

Amin Malekmohammadi

Aamir Malik

Satyanarayana Malladi

Aissa Manallah

Luis Mancera-Pascuals

Prasanta Mandal

Corey Manders

Nadarajah Manivannan

Gerald Manke

Qinghe Mao

Peter Marasco

Marian Marciniak

Richard Marino

Dan Marker

Ashot Markosyan

Daniel Marks

George Marmorino

Philip Marraccini

Valeria Marrocco

Manuel Martinez Corral

Fernando Martinez-Pinon

Alejandro Martinez-Rios

Koichi Maru

Jan Masajada

Atsunobu Masuno

Scott Mathews

Pierre Mathieu

Yoshihiko Matsuoka

Yuji Matsuura

Gary Matthews

Kent Mattsson

Tana Maurer

Stephen Maybank

Walton E. McBride, III

Donald McGaughey

James P. McGuire

lain McKenzie

Wayne McKinney

James McManamey

Paul McManamon

Kevin McNesby

Richard McWilliam

Alexander Meadway

Kate Medicus

Robert Meeks

P. Megret

Rekha Mehra

Ronald Meisels

Farid Melgani

Andrea Melloni 
Alexis Mendez

Rajin Mendis

Fernando Mendoza Santoyo

Aidong Meng

ChunNing Meng

Christoph Menke

P. Susthitha Menon

Joseph Meola

Juan Manuel Merlo

Luciano Mescia

Raed Mesleh

Wilhelmus Messelink

AJ Metcalf

Sheldon Meth

Ronald Meyers

Oleg Michailovich

Christian Micheloni

Michael Mielke

Emilia Mihaylova

Daniel Milanese

Miroslav Miler

Paul Miller

Predrag Milojkovic

Vladimir Minkovich

Vinod Mishra

Anupam Misra

Daniel Mittleman

Godai Miyaji

Katsuhiko Miyamoto

Andrzej Miziolek

Masato Mizukami

Vito Mocella

Waleed Mohammed

Arash Mokhtari

Vladimir Molchanov

Florian Moll

John Monnier

Joel B. Montgomery

Paul Montgomery

Ivo Montrosset

Jon Mooney

Andrew Moore

Duncan Moore

Richard Moore

Aldo Morales

Marissa E. Morales-Rodriguez

Alain Morand

Tim Morris

Ahmed Hisham Morshed

Christophe Moser

Martin Moskovits

Volodymyr Mosorov

Nasser Moustafa

Mikhail Mozerov

Bohdan Mroziewicz

Tingkui Mu

Weiqiang $\mathrm{Mu}$

Guido Mueller

Sudipta Mukhopadhyay

Linda Mullen
Arrate Munoz-Barrutia

J. Apolinar Munoz-Rodriguez

James F. Munro

Krishnamurthy Muralidhar

Paul Murphy

K. Murugesan

Ajay Nahata

Rajesh Nair

Mazen Nairat

Bikash Nakarmi

J. Nalaya

Yoshinori Namihira

Namu Narayanmurthy

Sivakumar Narayanswamy

Daniel Neal

Raluca Negres

Cristian Neipp

Drew V. Nelson

Sarik R. Nersisyan

Martin Neumann

Tuck Wah Ng

Dan Nguyen

$\mathrm{Na} \mathrm{Ni}$

Shahab Niazi

Jonathan Nichols

Josep Nicolas

Bai Nie

Feiping Nie

James Nikkel

Suwas Nikumb

John Noble

Takanori Nomura

Imama Noor

Gero Nootz

Rahman Nouroozi

V. Nourrit

Jiri Novak

Anu Nripanu

Paulo Nunes

Gualtiero Nunzi Conti

Sean O'Brien

Salah Obayya

Naoki Oda

Akira Ohte

Takashi Okamoto

Timothy Oldham

Vladimir Oliker

Arturo Olivares-Perez

Helder Oliveira

Jeffrey Oliver

Erik Olsson

Desmond Ong

Celestino Ordonez

Roberto Osellame

Miroslaw Ostaszewski

Wolfgang Osten

Yukitoshi Otani

Jose Manuel Oton

Peter Ott

Bing Ouyang
Sahin Ozdemir

Ibrahim Ozdur

Mehmet Ozturk

Jussi Paakkari

Alfonso Padilla-Vivanco

David Paglieroni

Radha Pal

Roman Palenichka

Bing Pan

JengShyang Pan

Jiahui Pan

Shilong Pan

Domenica Paoletti

Mandar Paranjape

Jae-Hyeung Park

Joong Mok Park

Kang Ryoung Park

Min Kyu Park

Sung-Bum Park

Unsang Park

Robert Parks

Inmaculada Pascual

Vittorio Passaro

Vishal Patel

Shibnath Pathak

Prasant Pattnaik

Peter Paul

Nicolaie Pavel

Nicolas Pavillon

Michal Pawlowski

Romuald Pawluczyk

Li Pei

Markus Peichl

Eli Peli

Paul Pellegrino

Stéphane Pelletier

Stefano Pelli

Danping Peng

PengChun Peng

Tao Peng

Xiang Peng

Xiaoyuan Peng

Yufeng Peng

Yunfeng Peng

Cesar Perciante

Matteo Perenzoni

Carlos Perez-Lopez

Felipe Perez-Rodriguez

Said Pertuz

Kara Peters

Petya Petkova

Nikolaos Petrellis

V.V. Petrov

Christian Pfluegl

Adam Phenis

Mark Phillips

Xiaodong $\mathrm{Pi}$

Daqing Piao

Pascal Picart

Massimo Piccardi 


\author{
Mark Pickering \\ H. Alan Pike \\ Patrick Pilarski \\ Hervé Piombini \\ Daniel Pipa \\ Mohammad Umar Piracha \\ Mahesh Pitchumani \\ Joe Pitman \\ Bruce Pixton \\ Aleksandra Pizurica \\ Peter Planinsic \\ Michael Player \\ William Plummer \\ Halina Podbielska \\ Ronald Polidan \\ Arup Polley \\ Pavel Polynkin \\ Juan Pomarico \\ Nithiroth Pornsuwancharoen \\ George Poropat \\ John N. Porter \\ Guy Potvin \\ John Powell \\ Andrea Prati \\ Stefan Preble \\ Alix Preston \\ Chrysanthe Preza \\ Brian Primeau \\ Christopher Progler \\ Francesco Prudenzano \\ Christof Pruss \\ Kostas Psannis \\ Jixiong $\mathrm{Pu}$ \\ Minhao Pu \\ Georg Pucker \\ Roberto Pugliese \\ Pulak Purkait \\ Feng Qi \\ Kemao Qian \\ Chengwei Qiu \\ Minghui Qiu \\ Alexander Quandt \\ Juan Antonio Quiroga \\ Thomas Raasch \\ Rabi Rabady \\ Balakrishnaiah Rachamadugu \\ Muhammad Rafiq Abuturab \\ Ashfaqur Rahman \\ M. Yasin Raja \\ K.B. Rajesh \\ Mohammad Rakib Uddin \\ Balakrishnan Ramamoorthy \\ Changhui Rao \\ Narayana Rao \\ Mani Maran Ratnam \\ Ileana Rau \\ Bernard Rauscher \\ Mark Ray \\ Mina Ray \\ Brendan Reagan
}

Patrick Reardon

David C. Redding

Tamer Refaat

Steffen Reichel

Daniel Reiley

Alfredo Remon

Chunxiao Ren

Deqing Ren

Yvon Renotte

Endre Repasi

Stefania Residori

Sergio Restaino

Vithyacharan Retnasamy

Phillip Reu

Juan Reyes-Gomez

Ahmed Reza

Francesco Riboli

Max Riedl

Eberhard Riedle

Kelly Risko

Davor Ristic

Grant Ritchie

Marco Riva

Nicolas Riviere

Maria Rizzi

Yong Ro

William Roach

Stefan Robila

Brian Robinson

Jeffrey Roblee

Rogéria Rocha Gonçalves

Carsten Rockstuhl

Ramon Rodriguez-Dagnino

Jose Rodriguez-Ramos

Günther Roelkens

John R. Rogers

Marek Romanowski

Murilo Romero

Michael Rooks

Peter Roos

Bakhtiar Rosdi

Armand Rosenberg

Alessandro Rossi

Yuri Rostovtsev

Gilles Roy

Jitendra Roy

Sukhdev Roy

Kuladeep Roy Chowdhury

Santiago Royo

Shuangchen Ruan

Alexander Rubenchik

Paul Ruffin

Cinzia Sada

Bouchta Sahraoui

D. R. Sahu

Partha Sahu

Prasant Sahu

Albert Salah

Leszek Salbut

Salvador Sales
Mohammed Salhi

Ertan Salik

Rosario Salvador-Palmer

Patrice Salzenstein

Alan Samuels

Juan Sanchez-Escobar

E. Sanchez-Nielsen

Xinzhu Sang

Gregory Sanger

Sucharita Sanyal

Scott Sarama

M. Iqbal Saripan

Wendy Sarney

Osami Sasaki

Jose Sasian

Angel Sauceda-Carvajal

Robinson Savarimuthu

Svetislav Savovic

Ediz Saykol

Ilyas Saytashev

Salvatore Scaglione

Gary S. Schajer

Robert J. Schalkoff

Staffan Schedin

Peter Schelkens

Giuseppe Schirripa Spagnolo

Leopold Schmetterer

Natalia Schmid

Greg Schmidt

Jason Schmidt

Markus Schmidt

Andreas Schmitt-Sody

Charles Schmuttenmaer

Stephen Schnelle

Marc Schnieper

Christian Schroer

Joel Schulman

Sylvain Schwartz

Craig Schwarze

Johannes Schwider

Jim Schwiegerling

Francesco Scotognella

Deron K. Scott

Angela Seddon

Chandra Sekhar Seelamantula

Patricia Segonds

Shankar Kumar Selvaraja

S. Sendhil Raja

Berardi Sensale-Rodriguez

Gowri Senthil

Paramasivam Senthilkumaran

Borja Sepulveda

Alexander Serebryakov

Joan Serra-Sagrista

Manuel Servin

Babak Seyed Aghazadeh

Dana Seyringer

Mehmet Sezgin

Wei Sha

Azad Shademan 
Mohammed Shahid

Marc Shalles

Alexander Shamray

Mingguang Shan

Vladimir Shandarov

Ajay Shankar

Mohan Shankar

Robert R. Shannon

K. Shantha Lakshmi

Peng Shao

Shuangyun Shao

Xiaopeng Shao

Asia Shapira

Jeffrey H. Shapiro

Kamalesh Sharma

Utkarsh Sharma

David L. Shealy

Bryan Shelton

Day-Fann Shen

Kun-Ching Shen

Jonathan Shephard

John Sheridan

Sergei Shestak

Vittala Shettigara

Daming Shi

Guangming Shi

Lilong Shi

Wei Shi

Xuli Shi

Tomoyoshi Shimobaba

Yehoshua Shimony

In Hee Shin

Eric Shirley

Mikhail Shlyagin

Mehrdad Shokooh-Saremi

Alireza Shooshtari

Raju Shrestha

Xuewen Shu

J. Paul Siebert

Thomas Siegfried

Raimo Silvennoinen

Donggyu Sim

Francesco Simoni

Rakesh Singh

Simranjit Singh

Sunil Singh

Tara Singh Kamal

Rahul Singhal

Saurabh Sinha

Indranil Sinharoy

Jean-Christophe Sinquin

Zvonimir Sipus

Lars Sjoqvist

Aris Skander

George Skidmore

Pierre Slangen

Frederic Smektala

Barry Smith

Charlene Smith

Leslie Smith
Grzegorz Soboń

Andre Sokolnikov

Amisha Somaiya

Manjunath Somayaji

Peter A.A.M. Somers

Jung-Young Son

Feng Song

Ho-Keun Song

Kyo Song

Limei Song

Qi Song

Xiaoyu Song

Yu Song

Yulin Song

Yakov Soskind

Amelia Carolina Sparavigna

Giorgio Speranza

Vasily Spirin

Pradeep Srinivasan

Uma Srinivasan

Anuj Srivastava

Craig Stacey

H. Stahl

David Statman

Elizabeth Steenbergen

Ove Steinvall

Milutin Stepic

Alvin Stern

Sebastian Stoebenau

David Stoltzmann

Bryan Stone

Elena Stoykova

Dimitra Stratis-Cullum

Donna Strickland

Marija Strojnik-Scholl

P. Strumillo

Guo-Dung Su

Hongjun Su

$\mathrm{Ke} \mathrm{Su}$

Peng Su

Yang Su

Yikai Su

Jose Suarez-Romero

Harish Subbaraman

S. Sudheer

Yusuke Sugano

Doug Suh

Iping Suhariadi

Xiubao Sui

Narayanan Unni Sujatha

An Sun

Dongsong Sun

Jian-feng Sun

Jinghua Sun

Kaiqiong Sun

Shih-Wei Sun

Xiankai Sun

Yunan Sun

Yuqiu Sun

Zheng Sun
Dirk Sutter

Ozgun Suzer

Orven F. Swenson

Mohamed A. Swillam

Tamas Sziranyi

Stefano Taccheo

Murat Tahtali

Enrique Tajahuerce

Nobuaki Takahashi

Yuzuru Takashima

Kei Takeya

Chee Hing Tan

Songxin Tan

Yannan Tan

Chen Tang

Dingyuan Tang

Xiahui Tang

XiaoHong Tang

Nelson Tansu

Guangming Tao

Jin Tao

Wenbing Tao

Konstantin Tapero

Jean-Philippe Tarel

Luiz Tarelho

Rao Tatavarti

James Taylor, Jr.

Lioudmila Tchvialeva

Chat Teeka

Jeremy Teichman

Vasily V. Temnov

Shuyun Teng

Fred Terry Jr.

Markus Testorf

Francis Theberge

James Theiler

Brian Thelen

Simon Thibault

Michael E. Thomas

Kevin Thompson

Thomas Thundat

Ping Tian

Xiang Tian

Ye Tian

Gil Tidhar

Ming-Chun Tien

Michael Tilleman

Brenda Timmerman

Antonio Ting

David Ting

Michael Ting

Bruno Tiribilli

Sanjiv Tiwari

Vidhu Tiwari

Vincent Toal

Hiroyuki Toda

Alexander Toet

David Tofsted

Takao Tomono

Stefanie Tompkins 


\author{
Behcet Toreyin \\ Italo Toselli \\ Csaba Toth \\ Alexis Tourapis \\ Sallie Townsend \\ Eric Tremblay \\ Gregoire Tremblay \\ Renu Tripathi \\ Marcelo Trivi \\ Johann Troles \\ Ute Troppenz \\ Mitch Troy \\ Du-Yin Tsai \\ Fuan Tsai \\ Tsung-Han Tsai \\ Kong Tsen \\ Edmond Turcu \\ Gonul Turhan-Sayan \\ J. Scott Tyo \\ Kenji Ueda \\ Wilfried Uhring \\ Luis Unzueta \\ Waclaw Urbanczyk \\ Boris Usievich \\ Alessandro Vaccari \\ Murukeshan Vadakke Matham \\ Juan A. Valles \\ Marc Van Droogenbroeck \\ Jurgen Van Erps \\ Roelof van Silfhout \\ Maurizio Vannoni \\ Javier Vargas \\ Fernando Vargas-Martin \\ Leo Varghese \\ Mario Vaz \\ Gleb Vdovin \\ Vijay Venkataraman \\ Vijayakumar Venugopal \\ Alicia Vera-Marquina \\ Antonio Verga \\ Jan Vermeiren \\ Narayanan Vijayakumar \\ Jose Vilas \\ Bram Vingerling \\ Matias Viotti \\ Enrico Virgilli \\ Jay Vizgaitis \\ Yvon Voisin \\ Benoit Vozel \\ Ashwin Wagadarikar \\ David Walker \\ Lingyu Wan \\ Wie Wan \\ Yongjian Wan \\ Allen Chi-Luen Wang \\ Chao Wang \\ Chinhua Wang \\ Chou-Chen Wang \\ Chuen-Ching Wang \\ Daihua Wang
}

Dayong Wang

Desong Wang

Gang Wang

Gang Wang

Guoyu Wang

Hai-feng Wang

Hao Wang

Huanqin Wang

Jiajun Wang

Jian Wang

Jianfei Wang

Jihua Wang

Jin Wang

Jun Wang

Ke Wang

Ke Wang

Lei Wang

Leiran Wang

Liang Wang

Mu-Chun Wang

Peng Wang

Pu Wang

Qi Wang

Qiang Wang

Qiang Wang

Qing Wang

Shuh-Yi Wang

Shuping Wang

Tingting Wang

Weiping Wang

Xingwei Wang

Xinhong Wang

Xu Wang

Yangli Wang

Yingxin Wang

Yong Wang

Yongchang Wang

Yonghong Wang

Yongtian Wang

Yuanqing Wang

Zhaoyang Wang

Zhi Wang

Zhi Wang

Zhicheng Wang

Stephen Warren-Smith

James Waterman

Alan Watson

David Webb

Jennifer Webb

Henk Weeber

Chia-Hung Wei

Xuetao Wei

Zhao Wei

Zhiyi Wei

Debra Weidanz

Shiuh-Ku Weng

Naoufel Werghi

Stefan Werling

Douglas H. Werner

Andreas Wetzig
John Whitaker

Jeffrey White

Lorne Whitehead

Justyna Widera

Rafał J. Wiglusz

David Wikner

Otakar Wilfert

Timothy D. Wilkinson

John A.R. Williams

David Wilson

Kim Winick

Mike Winter

Oliver Wirjadi

Withawat Withayachumnankul

Michael Withford

Nicole Wolffer

Chee Sun Won

Wai-on Wong

Lech Wosinski

Cameron Wright

Chongqing Wu

Jiaji Wu

Jian Wu

Jian Wei Wu

Po-Yi Wu

Sijin Wu

Yu Wu

Yue Wu

Zhenhai Wu

James Wyant

Susan Xavier

Haiyun Xia

Yong Xia

Zhixuan Xia

Tao Xian

Peng Xiang

Di Xiao

Jimin Xiao

Shilin Xiao

Tiqiao Xiao

Xiao Xiao

Xiao Xiao

Xifeng Xiao

Chongjin Xie

Dan Xie

Fang Xie

Feng Xie

Huimin Xie

J.J. Xie

Wei Xie

Wenjie Xie

Xin Xie

Jianguo Xin

Xiangjun Xin

Jian Xiong

Weihua Xiong

Dehui Xu

Dongjiang Xu

Juncheng $X u$

Lin Xu 
Nan Xu

Shixiang $X u$

Su Xu

Tingfa $X u$

Wen-Cheng Xu

Xiangfeng $\mathrm{Xu}$

Xiaoyin $\mathrm{Xu}$

Yong $\mathrm{Xu}$

Zhaowen $\mathrm{Xu}$

Qingsheng Xue

Yang Xuelin

Akira Yabe

Ichirou Yamaguchi

Masahiro Yamaguchi

Kenji Yamamoto

Manabu Yamamoto

Feng Yan

Peiguang Yan

Rongjin Yan

Aiying Yang

Bisheng Yang

Bo Yang

Fengjie Yang

Guanglin Yang

Guobiao Yang

Ho-Soon Yang

I-Chang Yang

Kun Tao Yang

Liming Yang

Lin Yang

Minghong Yang

Qi Yang

Ren Yang

Shan Yang

Shiyuan Yang

Shu Yang

Tzong-Jer Yang

Xu Yang

Yuan-Sen Yang

Yuanhong Yang

Sumio Yano

Jianping Yao

Xincheng Yao

Yao Yao

Leonid P. Yaroslavsky

Moh. Yasin

Chien-Hung Yeh

Pinghui Sophia Yeh

Sheng Lih Yeh

Seokwon Yeom

Allen $Y i$

Faliu Yi

Jonghoon Yi

Lilin Yi

Xingwen Yi

Alper Yildirim
August Yitzhak

Masayuki Yokota

Hidehiro Yonezawa

Hiroshi Yoshikawa

Shanhong You

Douglas Youmans

Garam Young

Rupert C. D. Young

Amr Yousef

Anthony $\mathrm{Yu}$

Changyuan $\mathrm{Yu}$

Chong-Shou Yu

Jianjun $Y u$

Mingbin $\mathrm{Yu}$

Wurong $Y u$

Yonglin $\mathrm{Yu}$

$\mathrm{Yu} \mathrm{Yu}$

Caojin Yuan

Hui Yuan

Xiao-Cong Yuan

Jia Yue

Heba Yuksel

Jong Pil Yun

Preecha Yupapin

Maria Yzuel

Saleem Zaidi

Marek Zajac

Jihad Zallat

Zhigang Zang

Hugo Zbinden

Jason Zeibel

Richard Zeleny

Herbert Zeman

Chao Zeng

Nan Zeng

Mourad Zghal

Guangtao Zhai

Pengwang Zhai

Bin Zhang

Caiyun Zhang

Changchun Zhang

Chunxi Zhang

Daming Zhang

Dong-Sheng Zhang

Hao Zhang

Li Zhang

Lin Zhang

Longyu Zhang

Min Zhang

Min Zhang

Ming Zhang

Peng Zhang

Rui Zhang

Shuqun Zhang

Song Zhang

Tong Zhang
Weizhong Zhang

Wentao Zhang

William Zhang

Xuming Zhang

Xusheng Zhang

Yan Zhang

Yonggang Zhang

Yuhua Zhang

Zhiyou Zhang

Zonghua Zhang

Chun-Liu Zhao

Chunliu Zhao

Chunyu Zhao

Jianhua Zhao

Lianjun Zhao

Lingjuan Zhao

Luming Zhao

Qing Zhao

Shengzhi Zhao

Yong Zhao

Yuanan Zhao

Yuxia Zhao

Zhengxin Zhao

Chuan-Tao Zheng

Jing Zheng

Ruitao Zheng

Shijie Zheng

Tianxiang Zheng

Xiaoping Zheng

Yuan Zheng

Dexing Zhong

Ping Zhong

Bo Zhou

Chuanhong Zhou

Guiyao Zhou

Haomin Zhou

Hui Zhou

Jian Zhou

Jingbo Zhou

Jun Zhou

Kaiming Zhou

Weihu Zhou

Yi Zhou

Yidong Zhou

Zhiping Zhou

Chunhua Zhu

Min Zhu

Ninghua Zhu

Xiaonong Zhu

Yinian Zhu

Zhu Qing Zhu

Chantal-Virginie Zint

David E. Zissa

Dimitrios Zografopoulos

Kyriakos Zoiros 